Historic, Archive Document

Do not assume content reflects current scientific knowledge, policies, or practices. 


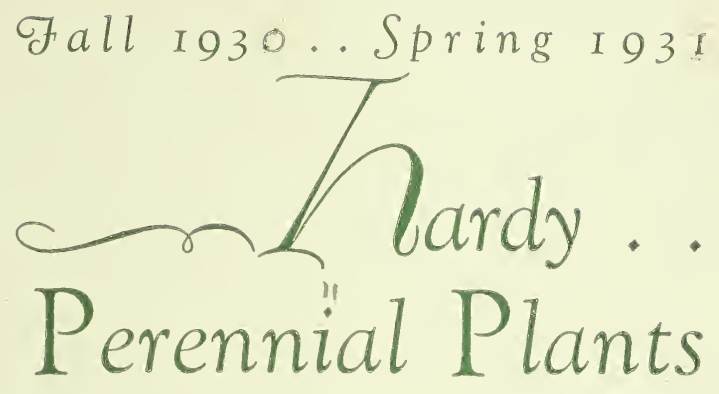

for

ROCK GARPEA

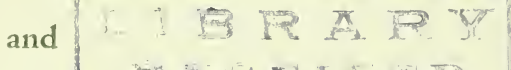

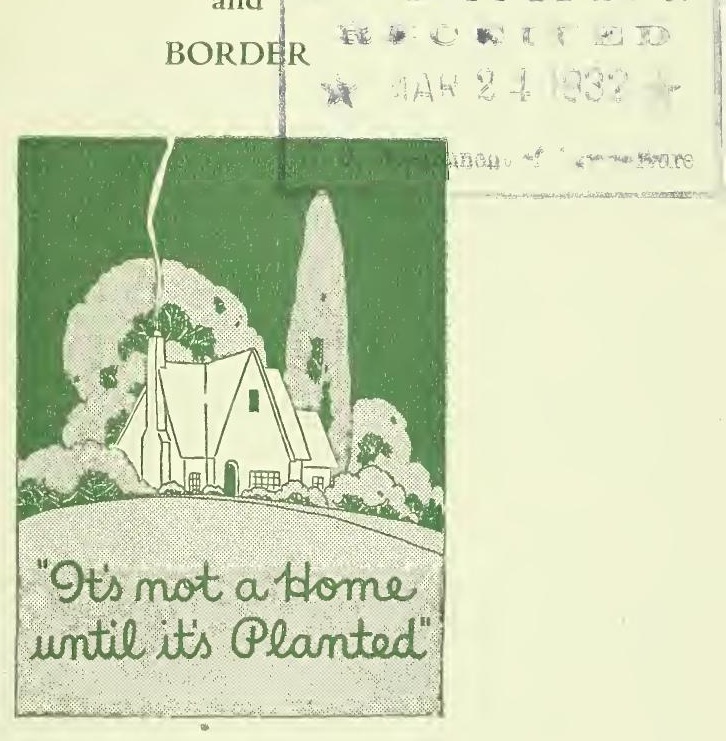

We Specialize in

OLD FASHIONED GARDEN FLOWERS AND ROCK GARDEN PLANTS

\section{Slemmons Gardens}

\section{Landscape Nurseries}

5256 Olentangy River Road

COLUMBUS, OHIO

Mailing Address, Worthington, O. LAwndale 3631 


\section{VISITORS WELCOME}

We cordially invite you to visit our display gardens. Our formal and rock gardens, pools and other garden features will be of interest to you and you will find new plants and new features being added constantly. We will gladly show you around any time of the day, evening or Sunday and stock will be dug for you any time except Sunday.

Member Ohio Nurserymen's Association.

Member Columbus Landscape Association. 


\section{TERMS OF SALE}

All orders are accepted subject to previous sale and condition of stock and under following terms and conditions.

\section{Prices}

All prices in this list are F. O. B. Worthing, ton, or Columbus. Free deliveries will be made in Columbus and vicinity on any orders amounting to $\$ 3.00$ or more. 1 dozen $25 \mathrm{c}$ plants of a kind $\$ 2.50$. 1 dozen $35 \mathrm{c}$ plants of a kind $\$ 3.50$. 6 plants of a kind at dozen rate.

\section{Shipping}

We will use our own judgment in effecting prompt and safe delivery of your plants unless shipping directions accompany order.

\section{Payments}

Cash must accompany orders unless customer is known to us and has an established credit.

\section{Planting Time}

Perennials may be planted from the last of March to the first of October, providing proper care in shading and watering is taken during the hot weather. However stock that must be packed and shipped can only be handled during the regular spring and fall seasons.

\section{Guarantee}

We guarantee every plant sold by us to be strong, vigorous and healthy and to deliver them to you in good condition. If for any reason you are not satisfied with your stock when it is received, advise us at once and we will gladly replace the item or refund the money.

We are constantly trying out new plants and varieties. As fast as they prove their worth they will be added to our list. 


\section{PRICE OF PLANTS}

ACHILLEA (Yarrow or Milfoil)

Ageratum, Yellow, dwarf................................. . .25

Filipendula, Yellow, $2 \mathrm{ft}$.................................. .25

Millefolium (Roseum) …................................ .25

Ptarmica, Boule de Neige, White...................... . .25

*Tomentosa, Yellow, dwarf............................... .25

ACONITUM (Monkshood)

Fischeri, Dwarf, blue........................................ .25

AGROSTEMMA (Mullin Pink)

Coronaria, Rose Champion................................ .25

*AJUGA (Bugle Weed)

Reptans rubra

ALTHEA ROSEA (Hollyhocks)

Double (Mixed Colors) ..................................... . .25

Single (Mixed Colors) ...................................... . .25

Allegheny's (Mixed) ...................................... .25

*ALYSSUM (Perennial Sweet Alyssum)

Argentum, Dwarf, shrubby, yellow................... . .25

Saxatile Compactum, Basket of gold................ .25

ANCHUSA (Bugloss)

Dropmore variety, Tall, blue ............................ .25

*Myosotidiflora, Dwarf, blue............................... . 35

ANTHEMIS (Marguerite)

Tinctoria Kelawyi, Yellow.

AQUILEGIA (Columbine)

*Alpina, Blue .................................................... .25

Canadensis, Native, red and yellow................. . .25

*Coerulea, Blue, long spurred.............................. .25

AQUILEGIA (Hybrids)

Mrs. Scott Elliot Hybrids.................................. . .25

Long Spurred Hybrids....................................... . 25

Extra fine doubles.......................................... .25

$\because$ ARABIS (Rock Cress)

Alpina, White

*ARMERIA (Statice. Thrift)

Cephalotes rubra, Deep pink............................. .35

Laucheana, Rosy Crimson, dwarf...................... . .35

Maritima, Pink …............................................. .25

ARTEMISIA (Sage Brush)

Lactiflora, White, scented, tall.......................... . .25

Silver King ................................................... .25

ASCLEPIAS (Butterfly Weed)

Tuberosa. Bright Orange, July...................... .25

ASTER (Perennial)

*Alpinus Goliath, Purple, 10 inches.................. .35

*Alpinus rubra, Rose, 10 inches.................... . .25

ASTER (Hardy)

Mauve Cushion, Dwarf............................... . 25

Fall Blooming, Tall (mixed colors) ….............. .25

* Rock Garden Plants.

1 Dozen 25c Plants of a Kind $\$ 2.50$.

1 Dozen 35c Plants of a Kind $\$ 3.50$.

6 Plants of a "Kind" at Dozen Rate. 
*AUBRETIA (Rock Cress)

Hybrids

BOLTONIA (False Chamomile)

Asteroides, White, 5 feet................................... .25

Latisquama, Pink, 4 feet..................................... . .25

BAPTISIA (False Indigo)

Australis, Blue

BELLIS (Double English Daisy)

Perenne, Pink and White.

BERGAMONT (Monarda)

Didyma, Red

BLEEDING HEART (See Dicentra)

BOCCONIA (Plume Poppy)

Cordata, 6 to $8 \mathrm{ft}$., white....

BUDDLEIA (Butterfly Bush)

Magnifica, Summer Lilac.

CALAMINTHA

Alpina, Purple, 6 in

*CAMPANULA (Alpine Varieties)

Carpatica, Blue ……........................................ .25

Canterbury Bells .......................................... . .25

Persicifolia (Peach Bell) ................................ .25

Rotundifolia (Blue Bell of Scotland) ................. .25

CARNATIONS (Hardy)

Mixed Colors

CENTAUREA (Perennial Cornflower)

Montana, Blue.

*CERASTIUM (Snow-In-Summer)

Tomentosum, White, silvery.

*CHEIRANTHUS (Siberian Wall Flower)

Allioni, Orange, dwarf...

CHRYSANTHEMUM (Hardy)

White, Yellow, Pink, Red and Bronze

Hardy Early Flowering.

CHRYSANTHEMUM MAXIMUM

(Shasta Daisy)

Various types

COREOPSIS (Harvest Moon)

Grandiflora, Yellow, all summer........................ .25

CRUCIANELLA

Stylosa

CONVALLARIA (Lily of the Valley)

CORONILLA (Crown Vetch)

Pink

CLEMATIS (Davidianna, Spring)

DELPHINIUM (Perennial Larkspur)

Belladonna, Light blue......

Bellamosa, Dark blue........................................... .25

Chinensis, Dwarf blue........................................... .25

Gold Medal Hybrids, 2 year.............................. . .35

Wrexham Strain Hybrids, 2 year..................... .50 
*DIANTHUS (Alpine Varieties)

Alpinus Allwoodi ................................ . 35

Caesius, Cheddar pink............................. . .25

Deltoides "Brilliant", Deep pink.................. .25

DIANTHUS (Border Varieties)

Barbatus, Sweet William, various colors......... .25

Hardy Carnations, Mixed.............................. . .25

*Plumarius, Old fashioned clove pink............. .25

Latifolius, Red ....................................... . 25

*DICENTRA (Bleeding Heart)

Eximea, Dwarf, long blooming, 1 year.......... .25

Spectabilis, 2 year................................... . 50

DICTAMNUS (Gas Plant) .......................... .50

DIGITALIS (Foxglove)

Shirley Hybrids, Best of all...................... .25

$\because$ ERINUS

Alpinus roseus, Dwarf alpine...................... . 35

EUPATORIUM (Hardy Ageratum)

Coelestinum, Blue, 2 feet....................... .25

ECHINACEA (Purple Coneflower)

Purpurpea

ECHINOPS (Thistle)

Ritro (Metallic blue)

ERYNGIUM (Sea Holly)

Amethystinum, Blue, winter bouquets............. .25

ERYSIMUM (Fairy Wallflower)

Pulchellum, Yellow

*FERNS (Hardy Dwarf)

Hardy native ferns for rock gardens and nat. uralizing ..........................................

We can supply strong native ferns in large quantities, four varieties, at special prices.

FUNKIA (Hosta, Plantain Lily)

Subcordata Grandiflora (White) ................... .25

Variegata (lavender) ............................... .25

GAILLARDIA (Blanket Flower)

Grandiflora, Red and yellow........

GEUM (Border)

Mrs. Bradshaw, Scarlet double..................... . .25

Lady Stratheden, Golden yellow.................... .25

GYPSOPHILA (Baby Breath)

Paniculata ........................................... . 25

*Repens, Dwarf, trailing, white..................... .25

GRASSES (Hardy)

Eulalia, Japonica ....................................... . .25

Eulalia, Variegata ................................. . .25

Phalaris, Ribbon grass............................ . 25

Festuce glauca ......................................... . 25 
HELENIUM (Helen's Flower)

Autumnale superbum, Rich yellow................ .25

Riverton Gem, Old gold............................ .25

HEMEROCALLIS (Day Lily)

Flava, Lemon lily.

*HEUCHERA (Coralbells)

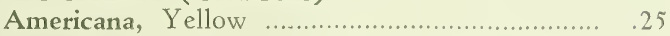

Sanguinea, Crimson, true........................... 35

HELIANTHUS (Hardy Sunflower)

Mollis, Yellow

HELIANTHEMUM (Rock or Sun Rose)

Choice mixed colors.

HEPATICA (Wood Anemone)

Coerulea

HESPERIS (Sweet Rocket)

Matronalis, Purple and white.

HIBISCUS (Mallow)

Red, white and pink

HOLLYHOCKS (See Alhtea Rosea)

IRIS GERMAN

40 named varieties, all standard varieties, wide range of color. Per clump....................... .25

Special mixtures, dozen.............................. 2.00

Special rate per hundred for mass planting.

\section{IRIS JAPANESE}

10 named varieties, a splendid group of both single and doubles.

\section{IBERIS (Hardy Candytuft)}

Sempervirens

Gilbraltorica

IRIS SIBIRICA (Siberian)

Blue

*LAVANDULA (Sweet Lavender)

Vera, True, fragrant blue.

*LEONTOPODIUM (Edelweiss)

Alpinum

LIATRIS (Kansas Gay Feather)

Scariosa, Purple ................................................. .35

Pycnostachya, Kansas Gayfeather....................... . .35

LATHYRUS (Everlasting Sweet Pea)

Pale Pink

Rosea

LINARIA (Toad Flax)

Kenworth Ivy

Dalmatica, Yellow

*LINUM (Flax)

Perenne, Blue, long blooming.

LOBELIA

Cardinalis, Cardinal Red 


\section{LUPINUS (Lupine)}

Hybrids, Mixed colors.

\section{LYCHNIS (Champion)}

Alpina, Rose pink....

Chalcedonica, Maltese Cross

LUNARIA (Honesty)

Biennis, Mixed

MATRICARIA (Feverfew)

White

MONARDA (Bergamot)

Didyma, Brilliant scarlet.

*MYOSOTIS (Forget-Me-Not)

Alpetris rosea ……........................................... .2

Palustris semperflorens, Blue............................ . 25

*NEPETA (Catnip)

Mussini, Dwarf, rich blue. True.................. .25

OENOTHERA (Evening Primrose)

Fraseri, Pale Yellow.............................................. .25

Youngi, Lemon yellow.................................. .25

\section{PEONIES}

Albert Crousse, Pink ......................................... 1.00

Felix Crousse, Bright red............................... 1.00

Mons. Jules Elie, Lilac pink............................ 1.00

Festiva Maxima, White...................................... .60

Karl Rosenfield, Red....................................... 1.25

Marguerite Gerard, Pink.................................. .60

Jeane Darce, Pink............................................. .60

Baroness Schroeder, White.............................. 1.25

\section{PENTSTEMON}

Barbatus Torreyi, Shell flower........................... .25

\section{PHLOX (Hardy Phlox)}

Africa, Carmen red............................................ .25

Beacon, Cherry red........................................... .25

Count Zeppelin, White, red eye........................ . .25

Fuerbrand, Orange scarlet................................ .25

Jules Sandeau, Watermelon pink ...................... . .25

Lord Raleigh, Purple mauve............................... . 25

Mia Ruys, White dwarf...................................... . .25

Mrs. Milly Van Hoboken, Pink....................... . .25

Rheinlander, Salmon pink............................... .25

Rijnstroom, Rose pink.................................... . .25

Thor, Salmon pink ....................................... .25

\section{PHLOX SUFFRUCTICOSA}

Miss Lingard, Waxy white, June................... .25

Miss Verboom, Rose pink................................ .25

\section{PHLOX (Alpine)}

Subulata alba, White........................................ .25

Subulata, Lilac ….......................................... . .25

Subulata, Rosea ..................................................... . .25

Fairy, Pale blue

Vivid, Brilliant pink............................................ .35 
PHYSALIS (Japanese Lantern Plant)

Francheti, Orange scarlet lanterns.

PHYSOSTEGIA (False Dragonhead)

Virginiana, Pink, late, 4 feet........................... .25

*Virginiana grandiflora "Vivid", Dwarf............ 35

PLATYCODON (Balloon Flower)

Grandiflora, Blue

Grandiflora alba, White.

*PRIMULA (Primrose)

Polyanthus veris hybrids, Bunch primrose....... . .35

Polyanthus, Improved .................................. .35

PLUMBAGO (Leadwort)

Larpentae, Blue

PYRETHRUM (Painted Daisy)

White, pink, crimson.

\section{POPPY (Orientale)}

Scarlet

Potted, strong plants ................................... .50

Spring, Salmon, pink and white potted.............. .50

Nudicaule (Iceland Poppy), White, yellow and orange

\section{POTENTILLA CINQUEFOIL}

Mixed colors

PACHYSANDRA, Jaq. Spurge.

$$
\$ 2.00 \text { for } 10-\$ 17.00 \text { per } 100 \text {. }
$$

\section{RANUNCULUS (Buttercup)}

Trailing Buttercup, Double yellow....

RUDBECKIA (Coneflower)

Newmanni (Black Eyed Susan) .................... .25

Purpurea, Purple coneflower............................ .25

Golden Glow, ......................................... .25

SALVIA (Meadow Sage)

Azurea, Blue sage from Rockies.................... .25

*SAPONARIA (Soapwort)

Ocymoides splendens, Dwarf, pink................ . .25

*SAXIFRAGA (Saxifrage)

Cordifolia, Megasea section, rose.................. .35

SCABIOSA (Hardy Pincushion Fower)

Caucasica, Lavender

\section{*SEDUM (Stone Crops)}

Acre, Dwarf, creeping, yellow bloom............ . 25

Acre album .............................................. .25

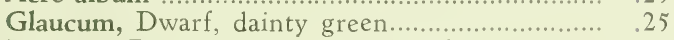

Lydium, Dainty evergreen, dwarf................... . 25

Pruniatum Fosterianum, one of best.

Sarmentosum, Yellow bloom. Rapid spreader .25

Sieboldi, Pink flowers in fall, best.................. .25

Spectabile, Erect, 1 foot, rose.......................... .25

Spectabile "Brilliant", Rich red..................... .25

Spurium coccineum, Showy red flowers, good .25

SPERGULA

Pilifera, 4 in., evergreen 


\section{*SEMPERVIVUM (Old Hen and Chickens)}

Alberta

Arachnoideum …............................................. .25

Globiferum …................................................. . .25

Tectorum ….................................................. . 25

*SILENE (Catchfly)

Alpestris, Dwarf, white, May........................... .25

Shafta, Dwarf, pink, fall................................. .25

*STACHYS (Lamb's Ears)

Lanata, Whitish, fuzzy leaves............................ . .25

STATICE (Sea Lavender)

Latifolia, Tiny blue flowers.............................. .25

STOKESIA (Cornflower Aster)

Laevis coreulea, large blue flowers................... .25

*THYMUS (Thyme)

Citriodorus, Lemon scented.............................. .25

Lanuginosus, Wooly leaved thyme.................... . .25

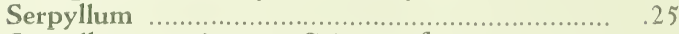

Serpyllum coccineum, Crimson flowers.............. .25

TRADESCANTIA (Spiderwort)

Virginiana, Blue flowers.................................... .25

TRITONIA (Red Hot Poker)

Pfitzeri

TROLLIUS

Hybrids

"TUNICA (Coat Flower)

Saxifraga, Prostate, rose flowers....................... .25

:VALERIANA (Valerian)

Coccinea, Old rose flowers................................. .25

Coccinea alba, White flowers.............................. . .25

Officinalis, Garden Heliotrope.......................... .25

*VERONICA (Speedwell)

Erica, Dilcate pink ............................................ . 35

Icana, Whitish leaves, blue flowers................... .35

Longifolia subsessilis, 2 feet, rich blue........... . 35

Marteni ….................................................... . .25

Teucrium, Blue, thrifty compact grower............ . 25

Teucrium rupestris, Dwarf blue, best............... . .35

Spicata, Erect, blue............................................. .25

*VIOLA

Cornuta, Mixed ................................................. $\quad .20$

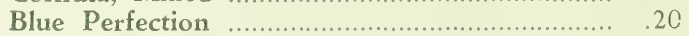

White Perfection .................................................. . 20

Lutea Splendens, Golden yellow........................ $\quad .20$

Papilio, Butterfly violet..................................... .20

Rose Queen .................................................... . .20

Apricot ..................................................... . 25

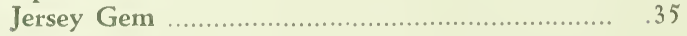

*YUCCA

YUCCA (Adam's Needles)

Filamentosa, Strong plants.............................. .50

VINCA (Trailing Myrtle)

Minor, 3 in. pots.. 
We grow a complete line of Nursery Stock, including Shrubs, Evergreens, Trees, Hedges, Vines, Bulbs, etc.

Our Landscape Service includes the making of Plans, Construction of Pools, Rock Gardens, Walks, Drives and Maintenance Work.

We have for sale Stone Benches, Bird Baths, Trellises, Fertilizer, Peat Moss, Grass Seed, Spray Materials, etc.

No longer do we confine our planting to the busy Fall and Spring seasons. Planting can be done at most any time of the year that the weather will permit. Shrubs and Evergreens may be planted any time during the summer, providing they are dug with a ball of dirt. Planting can also be done any time of the winter that the ground is not frozen.

We specialize in the winter moving of large trees. 


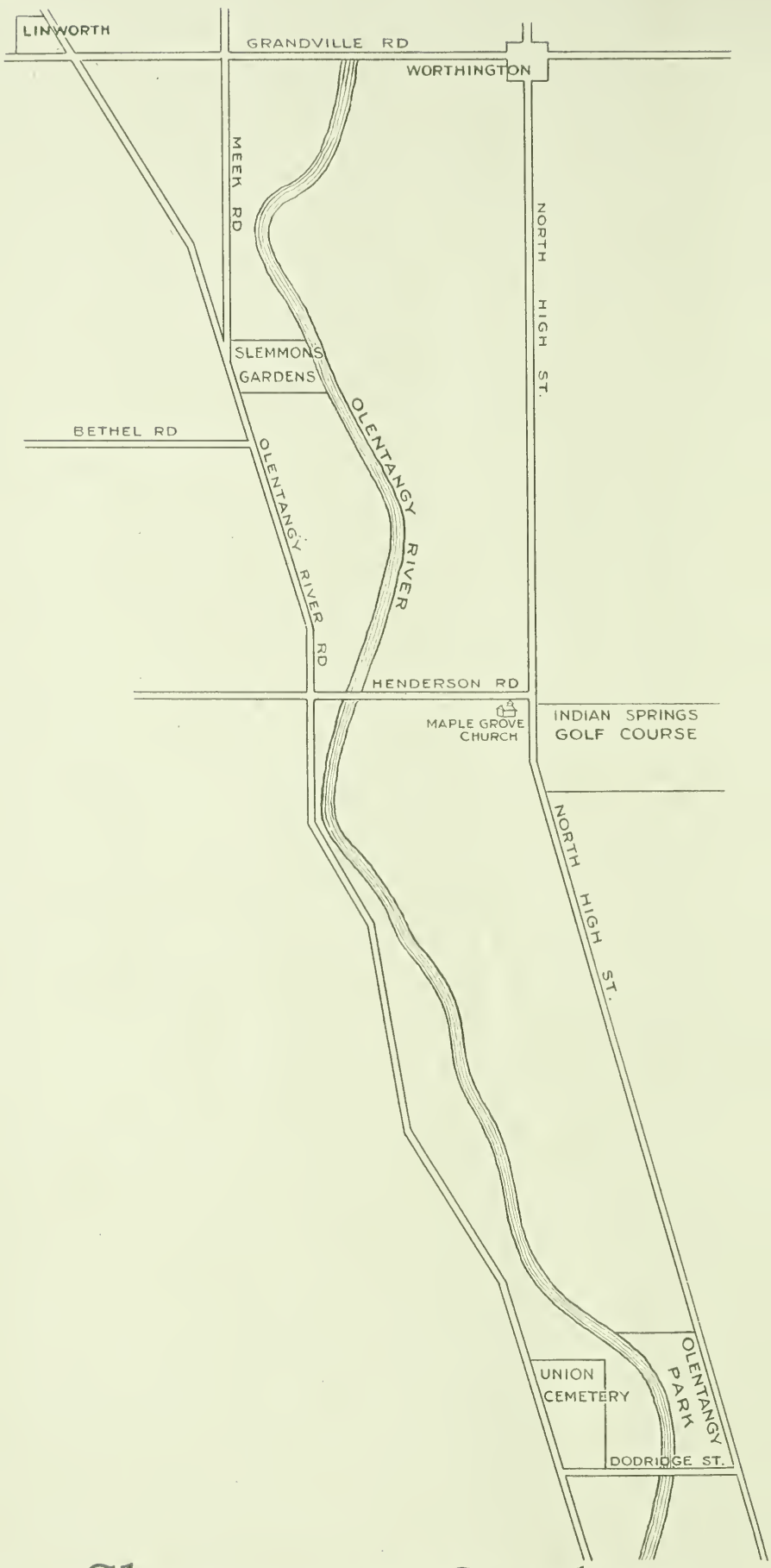

\section{Slemmons Gardens}

\section{Landscape Nurseries}

5256 Olentangy River Road

COLUMBUS, OHIO

Mailing Address, Worthington, O. LAwndale 3631 